\section{AB0096 ANALYSIS OF THE GENE EXPRESSION OF URAT-1, ABCG2, GLUT-9, OCT3, IL1B, TLR4 AND ALPK1 IN MONONUCLEAR CELLS OF PATIENTS WITH INTER- CRITICAL GOUT}

Natsuko Paniagua Díaz ${ }^{1}$, Laura Sanchez Chapul ${ }^{1}$, Carlos Aguilar Salinas² ${ }^{2}$, Alicia Ortega Mora ${ }^{1}$, Ambar López Macay. ${ }^{1}{ }^{1}$ Instituto Nacional de Rehabilitación, Neurociencias, Mexico City, Mexico; ${ }^{2}$ Instituto Nacional de Ciencias Medicas y Nutricion Salvador Subiran, Endocrinologia y Metabolismo, Mexico City, Mexico

Background: Gout is a common type of arthritis that has incresed in the world due to environmental and genetic factors that associate it with an altered metabolic state. In Mexico, overweight, obesisty, diabetes and metabolic syndrome have incresed in children and adult population, These alterations are associated with the development of gout in adults.

The gout is characterized by an inflammatory chronic process caused by the deposition of monosodium urate crystals (CUMs) in the joints. The recognition of these crystals by macrophages and neutrophils in the joint activates innate immunity. However, high levels of uric acid (UA) in intercritical phase could activate the expression of urate transporters and inflammation genes in other cell types such as monocytes and peripheral blood lymphocytes.

Objectives: The aim of this project was to analyze the changes in gene expres sion of SLC22A12, SLC2A9, SLC22A3, ABCG2, ALPK1, IL1ß, TLR4 in peripheral blood mononuclear cells (PBMC) of mexican patients with inter-critical gouty arthritis and controls without hyperuricemia $(\mathrm{HU})$ comparing it with their metabolic profile.

Methods: The determination of the biochemical parameters was performed by a serum - blood chemical test for uric acid, glucose, triglycerides, cholesterol, HDL, LDL and creatinine. through the use of UniCel DxC 600, Synchron Clinical System.

The total RNA of the PBMC cells was amplified by qRT-PCR using the GoTaq® 1Step RT-qPCR System real kit (Promega) and the Qiagen Rotor Gene Q kit, using specific primers. The gene expression analysis were performed by the $\triangle \mathrm{CT}$ and $\triangle \triangle \mathrm{CT}$ method and using GAPDH as a reference gene to compare the expression of urate transporter genes and inflammatory genes in the study groups.

The expression results were associated to clinical and biochemical parameters such as levels of uric acid, triglycerides, and weight. All statistical analyzes were performed with the SPSS program (IBM 22 version for Windows, SPSS, Inc., Chicago, IL, EU), using student's t-test or $\mathrm{U}$ Mann-Whitney test, for the normal and non-normal data, when $p^{*}<0.05$ or $p<{ }^{*} 0.01$.

Results: In this study we compared 24 patients with inter-critical gout and 26 controls without $\mathrm{HU}$. It was found that the expression of ABCG2 was higher in the patients and that the expression of OCT3 was higher in overweight people, regardless of whether they had gout or not compared with normal weigh people. The expression of ALPK1 was higher among patients and controls while the expression of TLR4 and IL1 $\beta$ was not different. The patients had a higher mean in the levels of UA, triglycerides, weight, age and hypertension, but lower HDL than the controls.

Conclusion: Patients with inter-critical gout maintain metabolic alterations associated with the metabolic syndrome although some of them are under treatment They are also characterized by having overexpression of ABCG2 and ALPK1. These conditions could favor a new attack of gout.

\section{REFERENCES}

[1] Dalbeth, N.; Merriman, T. R.; Stamp, L. K., Gout. Lancet 2016, 388 (10055), 2039-2052

[2] Merriman, T. R., An update on the genetic architecture of hyperuricemia and gout. Arthritis research \& therapy 2015, 17, 98

[3] Ko, A. M.; Tu, H. P.; Liu, T. T.; Chang, J. G.; Yuo, C. Y.; Chiang, S. L.; Chang, S. J.; Liu, Y. F.; Ko, A. M.; Lee, C. H.; Lee, C. P.; Chang, C. M.; Tsai, S. F.; Ko, Y. C., ALPK1 genetic regulation and risk in relation to gout. International journal of epidemiology 2013, 42 (2), 466-74.

Disclosure of Interests: None declared

DOI: 10.1136/annrheumdis-2019-eular.3901

\section{AB0097 NOVEL EX VIVOMODEL OF SEPTIC ARTHRITIS DEVELOPED TO IDENTIFY BIOMARKERS RELEASED UPON ARTICULAR INFECTION}

Kathryn McCall, Caroline Atherton, Neal L. Millar, Carl Goodyear, Tom Evans, lain Mcinnes. University of Glasgow, Institute of Infection, Immunity, and Inflammation, Glasgow, United Kingdom

Background: Septic arthritis (SA) is most commonly caused by infectious agents such as Staphylococcus aureus. Due to the morbidity and mortality associated with the disease, joint infections are treated as a medical emergency and therefore early diagnosis is essential. Unfortunately, no reliable biomarkers are available to assist diagnosis, beyond serum CRP and synovial fluid culture.

Objectives: To generate a novel ex vivo model of septic arthritis comprised of cartilage matrix, neutrophils and SA-associated Staph. aureus, to identify biomarkers released upon an inflammatory host response to infection.

Methods: Cartilage explants biopsied from human femoral heads were compared to chondrocyte disks differentiated from mesenchymal stem cells (MSC). MSC differentiation into chondrocytes under defined media and growth factor conditions was confirmed by IHC staining for collagen II on the disks. Explants and disks were infected for $48 \mathrm{~h}$ with $10^{6} \mathrm{cfu}$ Staph. aureus (SA patient derived). Structura damage was measured by glycosaminoglycan (GAG) release. Neutrophils were purified from health donor blood samples using the StemCell whole blood isolation kit, and $10^{6}$ cell were added in the final $4 \mathrm{~h}$ of bacterial infection. Disks were stained with eosin to visualise microscopic damage, CMFDA for viability and PI to identify cell death.

Results: Co-culture of MSC-derived chondrocyte disks with neutrophils or Staph aureus alone resulted in microscopically identifiable structural damage to the disk matrix. The addition of neutrophils in the final $4 \mathrm{~h}$ of the Staph. aureus co-cultures resulted in enhanced structural damage. Assessment of cellular viability revealed that both neutrophils and Staph. aureus alone induced cell death throughout the disk whilst the combination of both resulted in total abrogation of viable chondro cytes. Subsequent evaluation of GAG release, however, showed no clear differentiation between the conditions and as such this model was judged not to be sufficient for biomarker development. In comparison, femoral head cartilage explants studies demonstrated that Staph. aureus infection in this explant mode resulted in significant GAG release after $24 \mathrm{~h}$ and $48 \mathrm{~h}$, which was consistent between donors.

Conclusion: Cartilage explants provide a superior and more physiologically rele vant cartilage matrix model for the ex vivo analysis of septic arthritis. Staph. aureus infection resulted in reproducible damage to cartilage matrices that was not emulated in an MSC-derived chondrocyte disk system. These finding suggest that the femoral head cartilage explants are the optimal matrix model to identify biomarkers for joint infections.

Disclosure of Interests: Kathryn McCall: None declared, Caroline Atherton: None declared, Neal L Millar: None declared, Carl Goodyear Grant/research sup port from: AstraZeneca, BMS, Celgene, Janssen, MedAnnex, Pfizer and UCB, Speakers bureau: Abbvie, Tom Evans: None declared, lain Mclnnes Grant/ research support from: AstraZeneca, Celgene, Compugen, Novartis, Roche, UCB Pharma, Consultant for: AbbVie, Celgene, Galvani, Lilly, Novartis, Pfizer, UCB Pharma

DOI: 10.1136/annrheumdis-2019-eular.7501

\section{$\mathrm{AB} 0098$ \\ GENE EXPRESSION AND FUNCTIONAL COMPARISON BETWEEN MESENCHYMAL STEM CELLS FROM LATERAL AND MEDIAL CONDYLES OF KNEE OSTEOARTHRITIS PATIENTS}

Clara Sanjurjo Rodriguez ${ }^{1,2}$, Thomas Baboolal ${ }^{2,3,4}$, Agata Burska $^{2}$ Frederique Ponchel ${ }^{2}$, Jehan El-Jawhari ${ }^{2}$, Joseph Aderinto ${ }^{4}$, Owen Wall ${ }^{4}$ Hemant Pandit ${ }^{2,3,4}$, Dennis McGonagle ${ }^{2,3,4}$, Elena Jones ${ }^{2} .{ }^{1}$ Universidade da Coruña; INIBIC-CICA, CIBER-BBN, A Coruña, Spain; ${ }^{2}$ Leeds Institute of Rheumatic and Musculoskeletal Medicine, University of Leeds, Leeds, United Kingdom; ${ }^{3}$ NIHR Leeds Musculoskeletal Biomedical Research Unit, Leeds, United Kingdom; ${ }^{4}$ Leeds Teaching Hospitals NHS Trust, Leeds, United Kingdom

Background: Osteoarthritis $(\mathrm{OA})$ is the most common degenerative joint disor der, mainly afflicting the weight-bearing joints, and is the leading cause of physical disability worldwide. Despite the identified risk factors, the exact pathogenesis of osteoarthritis remains unclear (1). Formation of mesenchymal stem cells (MSC) clusters and their aberrant osteogenic differentiation in OA subchondra bone (SB) has been proposed as a key contributor to progression of OA in animal models (2) and in human hip OA (3). MSCs have a crucial role in joint repair but it remains unknown how OA severity affects MSC numbers or characteristics. Knee OA provides a good model to determine this as in knee OA, distribution of damage is usually asymmetrical and tends to be more severe in the medial (Med) compared to the lateral (Lat) compartment (4).

Objectives: The aim of this study was to determine whether there were differences in MSC numbers, topography and gene expression (GE) between Med and Lat femoral condyles of patients with knee OA

Methods: Condyles were obtained from OA patients that underwent total knee replacement ( $n=16$; UK Ethics Committee approval, 14/YH/0087). Decalcified samples were histologically evaluated for cartilage damage, bone sclerosis and CD271+ MSC distribution (2). MSCs were extracted from SB and sorted using the CD271+CD45- phenotype for GE analysis. Colony forming unit (CFU-F) (3), trilineage differentiation (5) and population doubling (PD) assays were performed. MSC numbers, topography and GE were compared between condyles.

Results: Med condyles presented significantly higher $(p<0.05)$ degree of cartilage damage (median OARSI score 20, range 15-20) compared to Lat condyles 